\title{
A functional inflammasome activation assay discriminates between genetically proven caps patients and patients with low penetrance NLRP3 variants
}

\author{
Nikolaus Rieber ${ }^{*}$, Alina Gavrilov, Theresa Endres, Dominik Hartl, Jasmin Kümmerle-Deschner \\ From 21st European Pediatric Rheumatology (PReS) Congress \\ Belgrade, Serbia. 17-21 September 2014
}

\section{Introduction}

The cryopyrin-associated periodic syndromes (CAPS) are characterized by recurrent episodes of systemic inflammation. CAPS is caused by mutations in the NLRP3 gene encoding cryopyrin, an important component of the NLRP3 inflammasome that activates caspase-1 resulting in inflammation by excessive production of IL- $1 \beta$ and others. A diagnostic dilemma is often encountered in patients with unspecific inflammatory symptoms like fatigue, muscle pain, arthralgia or slight hearing loss and low penetrance variants in NLRP3 / CIAS with an inconsistent clinical phenotype. The analysis of IL- $1 \beta$ in the serum did not prove to be a valid diagnostic test in these individuals.

\section{Objectives}

In this study we sought to investigate, if a functional inflammasome activation assay discriminates between genetically proven CAPS patients, patients with low penetrance NLRP3 variants and healthy controls.

\section{Methods}

The study population consisted of 16 patients with genetically proven Muckle-Wells syndrome, 9 patients with low penetrance NLRP3 variants (V198M, Q703K and E627G) and 14 healthy controls. Concentrations of IL-1 $\beta$, IL-1 $\alpha$, IL-18, and Caspase- 1 were quantified in cell culture supernatants after inflammasome stimulation with LPS and LPS + ATP for several timepoints.

\section{Results}

After 4h of LPS stimulation, secretion of NLRP3 inflammasome products (IL-1 $\beta$, IL-1 $\alpha$, IL-18) and Caspase-1 were potently increased in MWS patients, whereas there was no increase in low penetrance NLRP3 variants and healthy controls (for IL- $1 \beta \mathrm{p}<0.001$ and $\mathrm{p}<0.001$, respectively). Minor differences were still detected at later timepoints and for LPS + ATP stimulation.

\section{Conclusion}

Our functional inflammasome activation assay discriminates between genetically proven CAPS patients and patients with low penetrance NLRP3 variants. This assay might add to the decision, which individuals presumably benefit from an anti-IL-1 therapy.

\section{Disclosure of interest}

N. Rieber Grant / Research Support from: Novartis Research Grant, A. Gavrilov: None declared., T. Endres: None declared., D. Hartl: None declared., J. KümmerleDeschner Grant / Research Support from: Novartis Research Grant.

Published: 17 September 2014

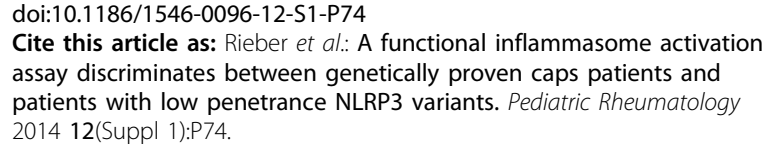

Cite this article as: Rieber et al:: A functional inflammasome activation assay discriminates between genetically proven caps patients and patients with low penetrance NLRP3 variants. Pediatric Rheumatology 2014 12(Suppl 1):P74 